\title{
Modeling and Identification of Supply Chains on Subspace Basis
}

\author{
T. Vadvári ${ }^{1}$, P. Várlaki ${ }^{1}$ \\ ${ }^{1}$ Széchenyi István University, System Theory Laboratory \\ Egyetem tér 1., 9026 Győr, Hungary \\ E-mail: varlaki@sze.hu
}

Abstract: Logistical processes play crucial role in many systems where the resources as well as tasks related to them are to be organized or scheduled in order to increase the throughput of the system, thus the rate of servicing demands. In this paper let us focus on loading systems and supply chains which represent a specific type of logistical processes. In order to increase the throughput of a loading system, delays caused by its certain subsystems are to be minimized. In our previous studies we have already shown how loading systems can be identified on subspace basis and how the relation between its certain parameters can be characterized. In contrast this paper is aimed to show through examples how system delays affect the efficiency of subspace based identification applied on loading systems.

Keywords: Subspace identification, queuing models, supply chains, modeling

\section{Introduction}

Supply chains as a specific category in logistics might be represented as a network of nodes representing facilities that perform some actions related first of all to manufacturing, transportation and distribution of products. Depending on the participant of the supply chain such a network may consist of many interconnected nodes, with specific characteristics [1]. Thus, the behavior of a complex supply chain may depend on many factors. The topology of the network may change as well especially when a facility becomes unable to fulfill its function due to some reasons. The authors in [2] for example deal with so called integrated supply chain optimization problems where the locations of facilities, customer allocations, etc. are considered as well when facilities are subject to disruption risks.

In order to identify these factors as well as their impact on the whole chain, modeling and identification of the system has to be performed. As in many fields also in logistics modeling and identification approaches play significant role especially when accurate models of complex logistical processes (LP) have to be designed. Authors in [3] consider 
queuing models to compute the response time for the delivery of items. Proper supply chain models may be helpful to predict various features related to the modeled system, such as the response time or in case of supply chains the delivery cycle time, customer order path (related to time spent in different channels), etc. In addition a good model may be helpful also to identify critical nodes in the chain and help to improve its reliability by changing the topology of the supply chain network. In case when the inner structure of the system is unknown (for instance the concrete service strategy and other internal mechanisms) the modeling and identification can be performed only based on the available measurement input-output data, which means only black box like modeling and identification methods are utilized. In the literature many models (as for instance scheduling, transportation planning, flow-shop sequencing problem) related to logistic systems are based on the fuzzy set and fuzzy control theory, statistics or their combination [4][5][6][7][8][9].

During our previous studies we have investigated state space models together with subspace based identification to model and identify supply chains or loading systems and characterize the dependency between its certain factors [10]. A framework to promote the better understanding of supply chain performance measurement and metrics can be followed for example in [11]. In this paper let us focus on the impact of delays on the accuracy of the identified model by considering state space models and subspace identification techniques.

Many times it is difficult to find a proper mathematical model which would suitable approximate the behavior of the observed logistical process even if the identification of the system is considered locally. However subspace identification techniques combined with tensor product transformation seem to be promising to model complex logistical processes based on input-output data. In this case there is no need for an explicit model parametrization, which is a rather complicated matter for multi-output linear systems [12]. Based on given input-output pairs (which provide useful information about the unknown system) the discrete time, linear, time-invariant state space model of a logistical system (see later in section 3) will be identified by using subspace techniques.

Depending on the knowledge about the modeled system a broad range of solutions can be utilized. Since complex logistical systems are non-linear MIMO systems and are influenced by many parameters their modeling is not a trivial task. Many methods have been proposed to deal with multi-input, multi-output systems in the literature. Perhaps the most popular tool in this topic is the linear parameter varying (LPV) structure by which non-linear systems can be modeled and controlled on the basis of linear control theories. Furthermore, the most recent results of the numerical algebra, such as the higher order singular value decomposition and the related tensor product transformation (making connection between LPV models and higher order tensors) offer promising tools to bridge heuristic and analytic approaches. In such a joint framework besides analytic description of the system the expert knowledge can be considered, as well. This may further improve the effectiveness and extend the applicability of the related methods [13]-[16]. 
The paper is organized as follows: Section 2 gives a brief overview of subspace identification for deterministic case. In Section 3.1 and 3.2 examples are reported showing the effect of delays in supply chains on the accuracy of the identified model. Finally conclusions are reported.

\section{Overview on Subspace Identification of LTI Systems}

Before turning the focus onto modeling loading systems and related delays, let us give a brief description on how subspace identification techniques may be used to identify linear time invariant (LTI) vertex models in the parameter space. Let us assume that the local behavior of the logistical system is deterministic, thus it can be described in the well known state space form as follows:

$$
\begin{gathered}
\mathbf{x}_{k+1}=\mathbf{A} \mathbf{x}_{k}+\mathbf{B} \mathbf{u}_{k} \\
\mathbf{y}_{k}=\mathbf{C} \mathbf{x}_{k}+\mathbf{D} \mathbf{u}_{k},
\end{gathered}
$$

where $\mathbf{x}_{k} \in \mathbb{R}^{n}$ stands for the state vector, $\mathbf{u}_{k}$ and $\mathbf{y}_{k}$ represent the input and output vector respectively at time $k T$, where $T$ stands for the sampling time. The goal is to find the model matrices $\mathbf{A}, \mathbf{B}, \mathbf{C}$ and $\mathbf{D}$ based on input-output pairs. As described in [17] let us first arrange the input-output pairs into so called Hankel matrices (reflecting the history of our input-output data):

$$
\begin{gathered}
\mathbf{U}_{1 \mid i}=\left[\begin{array}{cccc}
\mathbf{u}_{1} & \mathbf{u}_{2} & \ldots & \mathbf{u}_{j} \\
\mathbf{u}_{2} & \mathbf{u}_{3} & \ldots & \mathbf{u}_{j-1} \\
\vdots & \vdots & \ldots & \vdots \\
\mathbf{u}_{i} & \mathbf{u}_{i+1} & \ldots & \mathbf{u}_{j+i-1}
\end{array}\right], \\
\mathbf{Y}_{1 \mid i}=\left[\begin{array}{cccc}
\mathbf{y}_{1} & \mathbf{y}_{2} & \ldots & \mathbf{y}_{j} \\
\mathbf{y}_{2} & \mathbf{y}_{3} & \ldots & \mathbf{y}_{j-1} \\
\vdots & \vdots & \ldots & \vdots \\
\mathbf{y}_{i} & \mathbf{y}_{i+1} & \ldots & \mathbf{y}_{j+i-1}
\end{array}\right],
\end{gathered}
$$

where, $i$ stands for the number of block rows (should be selected to be larger than the maximum order $n$ of the system) and $j$ denotes the number of columns, which in case of using all data samples is equal to $N-i$, where $N$ represents the number of all input-output samples. Furthermore, let the history of states (unknown) to be estimated encode as follows:

$$
\mathbf{X}_{i}=\left[\begin{array}{llll}
\mathbf{x}_{i} & \mathbf{x}_{i+1} & \ldots & \mathbf{x}_{i+j-1}
\end{array}\right]
$$


It can be recognized from (2) that all row vectors in $\mathbf{Y}_{1 \mid i}$ are in the vector space determined by the union of row space of $\mathbf{X}_{i}$ and $\mathbf{U}_{1 \mid i}$. Let us assume that the intersection of row space of $\mathbf{X}_{i}$ and $\mathbf{U}_{1 \mid i}$ is empty. The most simple alternative for estimating $\mathbf{X}_{i}$ (up to a constant multiple $\mathbf{C}$ ) is to project the row space of $\mathbf{Y}_{i}$ onto orthogonal complement of the row space of $\mathbf{U}_{1 \mid i}$. The elements of $\mathbf{Y}_{i}$ can be expressed with the help of the extended observability matrix $\boldsymbol{\Gamma}_{\mathbf{i}}$ and lower block triangular Toeplitz matrix $\mathbf{H}_{i}$ form as follows [17]:

$$
\mathbf{Y}_{1 \mid i}=\Gamma_{\mathbf{i}} \mathbf{X}_{\mathbf{1}}+\mathbf{H}_{\mathbf{i}} \mathbf{U}_{\mathbf{1} \mid \mathbf{i}}
$$

where

$$
\boldsymbol{\Gamma}_{\mathbf{i}}=\left[\begin{array}{llll}
\mathbf{C} & \mathbf{C A} & \ldots & \mathbf{C A}^{i-1}
\end{array}\right]^{\top}
$$

and

$$
\mathbf{H}_{\mathbf{i}}=\left[\begin{array}{ccccc}
\mathbf{D} & 0 & 0 & \ldots & 0 \\
\mathbf{C B} & \mathbf{D} & 0 & \ldots & 0 \\
\mathbf{C A B} & \mathbf{C B} & \mathbf{D} & \ldots & 0 \\
\mathbf{C A}^{i-2} \mathbf{B} & \mathbf{C A}^{i-3} \mathbf{B} & \ldots & \mathbf{C B} & \mathbf{D}
\end{array}\right] .
$$

By substituting recursively into (1) we can express the state sequence $\mathbf{X}_{i+1}$ as follows:

$$
\mathbf{X}_{i+1}=\mathbf{A}^{i} \mathbf{X}_{1}+\boldsymbol{\Delta}_{i} \mathbf{U}_{1 \mid i},
$$

where

$$
\boldsymbol{\Delta}_{i}=\left[\begin{array}{lllll}
\mathbf{A}^{i-1} \mathbf{B} & \mathbf{A}^{i-2} \mathbf{B} & \ldots & \mathbf{A B} & \mathbf{B}
\end{array}\right]
$$

stands for the reversed extended controllability matrix [17]. From (6) the state sequence $\mathbf{X}_{1}$ can be expressed as:

$$
\mathbf{X}_{1}=\Gamma_{i}^{*} \mathbf{Y}_{1 \mid i}-\Gamma^{*}{ }_{i} \mathbf{H}_{i} \mathbf{U}_{1 \mid i},
$$

where $*$ denotes conjugate transpose of the matrix. By substituting (11) into (9) we obtain:

$$
\mathbf{X}_{i+1}=\mathbf{A}^{i} \boldsymbol{\Gamma}^{*}{ }_{i} \mathbf{Y}_{1 \mid i}-\mathbf{A}^{i} \boldsymbol{\Gamma}^{*}{ }_{i} \mathbf{H}_{i} \mathbf{U}_{1 \mid i}+\boldsymbol{\Delta}_{i} \mathbf{U}_{1 \mid i} .
$$

Let us express $\mathbf{X}_{i+1}$ as the sum of two matrices, where one of the matrices contains only the input-output values, i.e.

$$
\mathbf{X}_{i+1}=\mathbf{L}_{i} \mathbf{W}_{1 \mid i}
$$

where

$$
\mathbf{L}_{i}=\left[\boldsymbol{\Delta}_{i}-\mathbf{A}^{i} \boldsymbol{\Gamma}^{*}{ }_{i} \mathbf{H}_{i} \quad \mathbf{A}^{i} \boldsymbol{\Gamma}^{*}{ }_{i}\right]
$$

and

$$
\mathbf{W}_{1 \mid i}=\left[\begin{array}{ll}
\mathbf{U}_{1 \mid i} & \mathbf{Y}_{1 \mid i}
\end{array}\right]^{\top}
$$

Since based on (6)

$$
\mathbf{Y}_{i+1 \mid 2 i}=\boldsymbol{\Gamma}_{\mathbf{i}} \mathbf{X}_{\mathbf{i}+\mathbf{1}}+\mathbf{H}_{\mathbf{i}} \mathbf{U}_{\mathbf{i}+\mathbf{1} \mid \mathbf{2} \mathbf{i}}=\boldsymbol{\Gamma}_{\mathbf{i}} \mathbf{L}_{\mathbf{i}} \mathbf{W}_{\mathbf{1} \mid \mathbf{i}}+\mathbf{H}_{\mathbf{i}} \mathbf{U}_{\mathbf{i}+\mathbf{1} \mid \mathbf{2} \mathbf{i}}
$$

Let us now project $\mathbf{Y}_{i+1 \mid 2 i}$ onto orthogonal complement of $\mathbf{U}_{i+1 \mid 2 i}$. Since the projection of $\mathbf{H}_{i} \mathbf{U}_{i+1 \mid 2 i}$ onto its orthogonal complement is empty subspace we obtain [17]: 


$$
\begin{gathered}
\mathbf{Y}_{i+1,2 i} / \mathbf{U}_{i+1,2 i}^{\perp}=\boldsymbol{\Gamma}_{i} \mathbf{L}_{i} \mathbf{W}_{1 \mid i} / \mathbf{U}_{i+1,2 i}^{\perp} \\
\left(\mathbf{Y}_{i+1,2 i} / \mathbf{U}_{i+1,2 i}^{\perp}\right)\left(\mathbf{W}_{1 \mid i} / \mathbf{U}_{i+1,2 i}^{\perp}\right)^{-1}=\boldsymbol{\Gamma}_{i} \mathbf{L}_{i}, \\
\underbrace{\left(\mathbf{Y}_{i+1,2 i} / \mathbf{U}_{i+1,2 i}^{\perp}\right)\left(\mathbf{W}_{1 \mid i} / \mathbf{U}_{i+1,2 i}^{\perp}\right)^{-1} \mathbf{W}_{1 \mid i}}_{\mathbf{O}_{i+1}}=\boldsymbol{\Gamma}_{i} \underbrace{\mathbf{L}_{i} \mathbf{W}_{1 \mid i}}_{\mathbf{X}_{i+1}}, \\
\mathbf{O}_{i+1}=\boldsymbol{\Gamma}_{i} \mathbf{X}_{i+1}
\end{gathered}
$$

where $\mathbf{U}^{\perp}$ denotes the orthogonal complement of the row space of $\mathbf{U}$. Let us investigate the structure of $\mathbf{O}_{i+1}$. Based on (7) and (5) it can be expressed as:

$$
\mathbf{O}_{i+1}=\left[\begin{array}{llll}
\mathbf{C} & \mathbf{C A} & \ldots & \mathbf{C A}^{i-1}
\end{array}\right]^{\top}\left[\begin{array}{llll}
\mathbf{x}_{i+1} & \mathbf{x}_{i+2} & \ldots & \mathbf{x}_{i+j}
\end{array}\right] .
$$

Based on (21) the rank of $\mathbf{O}_{i+1}$ equals to the rank of the state sequence matrix $\mathbf{X}_{i+1}$. Equivalently, the dimensionality of the state vector $\mathbf{x}$ equals to the dimensionality of $\mathbf{O}_{i+1}$. The rank of $\mathbf{O}_{i+1}$ can be determined by singular value decomposition (SVD) as follows[17]:

$$
\begin{gathered}
\mathbf{O}_{i+1}=\mathbf{U}_{1} \mathbf{S}_{1} \mathbf{V}_{1} \\
\boldsymbol{\Gamma}_{i} \mathbf{X}_{i+1}=\mathbf{U}_{1} \mathbf{S}_{1}^{1 / 2} \mathbf{T T}^{-1} \mathbf{S}_{1}^{1 / 2} \mathbf{V}_{1},
\end{gathered}
$$

where $\mathbf{T}$ is an arbitrary invertible square matrix representing a similarity transformation.

$$
\begin{gathered}
\mathbf{X}_{i+1}=\mathbf{T}^{-1} \mathbf{S}_{1}^{1 / 2} \mathbf{V}_{1} \\
\widetilde{\mathbf{X}}_{i+1}=\mathbf{S}_{1}^{1 / 2} \mathbf{V}_{1}
\end{gathered}
$$

The system matrix can be estimated in the least squares sense from the following set of equations:

$$
\left[\begin{array}{l}
\widetilde{\mathbf{X}}_{i+2} \\
\mathbf{Y}_{i+1}
\end{array}\right]=\left[\begin{array}{cc}
\widetilde{\mathbf{A}} & \widetilde{\mathbf{B}} \\
\widetilde{\mathbf{C}} & \widetilde{\mathbf{D}}
\end{array}\right]\left[\begin{array}{l}
\widetilde{\mathbf{X}}_{i+1} \\
\mathbf{U}_{i+1}
\end{array}\right],
$$

where $\mathbf{U}_{i+1}$ and $\mathbf{Y}_{i+1}$ are input and output block Hankel matrices, respectively having one block row.

\section{Modeling Supply Chains on Subspace Basis}

In this section let us show through examples how supply chains can be modeled and identified on subspace bases. Furthermore let us also show through examples how the response times in the supply chain influence the identified model. The simulations have been performed by sampling interval $T=1[\mathrm{sec}]$. 


\subsection{Example 1}

In this example transporting vehicles are loaded according to incoming demands in order to transport goods from warehouse located at A to a given destination B. To service a demand a free vehicle is needed to transport goods, loading machines are required to load the vehicle at A and unload it after its arrival at B. If the unloading process is completed the vehicle may return to the warehouse and wait for new incoming demands. The block diagram of the system used to generate simulation data can be seen in Fig. 1 while the parameters together with their actual values can be followed in Table 1.

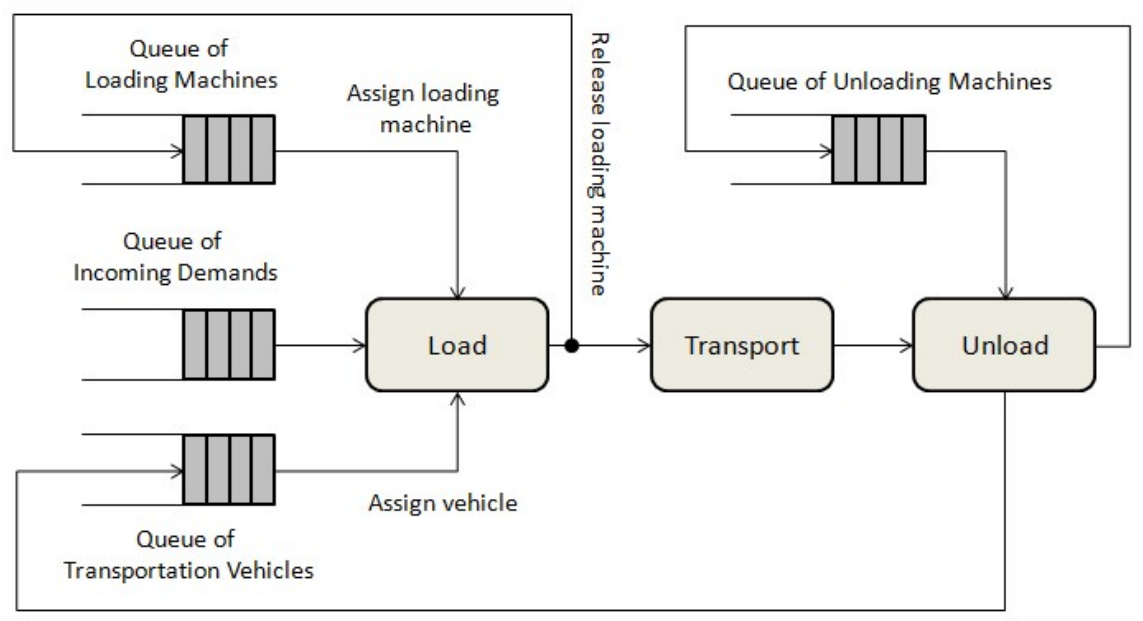

Release vehicle

Figure 1. The architecture of the system designed to generate simulation data

Let us consider input-output pairs represented by the average waiting time of demands in the queue of demands as input $u_{1}$ and the average service time of demands as output $y_{1}$. Let us identify the deterministic state space model describing the relationship between these two mentioned system parameters by using the above described subspace identification technique (see 2 for more details about subspace identification).

The input $u_{1}$ can be followed in Fig. 2 while the corresponding simulated output (representing the measured data) together with the output of the estimated model are depicted in Fig. 3. The model estimation was performed based on 800 input-output data pairs. During the verification 1600 data pairs have been considered. The matrices of the identified state space model are as follows: The estimated system matrix: 
Table 1. Setup of system parameters to generate simulation data

\begin{tabular}{l||l}
\hline Parameter & Value \\
\hline \hline Number of loading machines & 10 \\
Number of vehicles & 10 \\
Number of unloading machines & 3 \\
Queue length of loading machines & 40 \\
Queue length of unloading machines & 40 \\
Queue length of vehicles waiting for loading & 40 \\
Queue length of incoming demands & 50 \\
Queue length of arrived vehicles & 40 \\
Loading time & Exponential distribution with mean 10 \\
Unloading time & Exponential distribution with mean 10 \\
Transport time & Exponential distribution with mean 10 \\
Return time of vehicles & Exponential distribution with mean 10 \\
Incoming rate of demands & Exponential distribution with mean 1 \\
\hline
\end{tabular}

$$
\mathbf{A}=\left[\begin{array}{rrrrrrr}
0.65029 & -0.1937 & 0.28359 & 0.17257 & -0.17277 & -0.0097291 & 0.078612 \\
-0.3566 & 0.68973 & 0.29488 & 0.36481 & -0.2482 & -0.064809 & 0.098057 \\
-0.36349 & -0.39037 & -0.27047 & 0.34423 & 0.22771 & -0.17696 & 0.055399 \\
0.038496 & -0.11669 & -0.57838 & -0.13653 & -0.8574 & -0.42366 & -0.21459 \\
0.15813 & 0.31372 & -0.25643 & 0.21981 & 0.28052 & -0.52284 & -0.11093 \\
0.094546 & -0.23733 & 0.00031895 & 0.43292 & 0.068304 & -0.35473 & -0.5905 \\
-0.082549 & -0.0076265 & 0.29503 & -0.21588 & 0.042108 & -0.28678 & -0.61157
\end{array}\right]
$$

The estimated input matrix:

$$
\mathbf{B}=\left[\begin{array}{lllllll}
-0.17348 & -0.33904 & -1.1252 & 2.1473 & 0.78504 & 2.2637 & 4.2016
\end{array}\right]^{T}
$$

The estimated output matrix:

$$
\mathbf{C}=\left[\begin{array}{lllllll}
39.62 & -9.8396 & 2.9335 & 1.5995 & -3.1529 & 3.2417 & -0.16641
\end{array}\right]
$$

The initial state of the estimated system:

$$
\mathbf{X}(\mathbf{0})=\left[\begin{array}{lllllll}
12.427 & 23.417 & 82.997 & -155.83 & -74.708 & -170.02 & -361.04
\end{array}\right]^{T}
$$


T. Vadvári and P. Várlaki - Acta Technica Jaurinensis, Vol.10., No.2., pp. 84-98, 2017

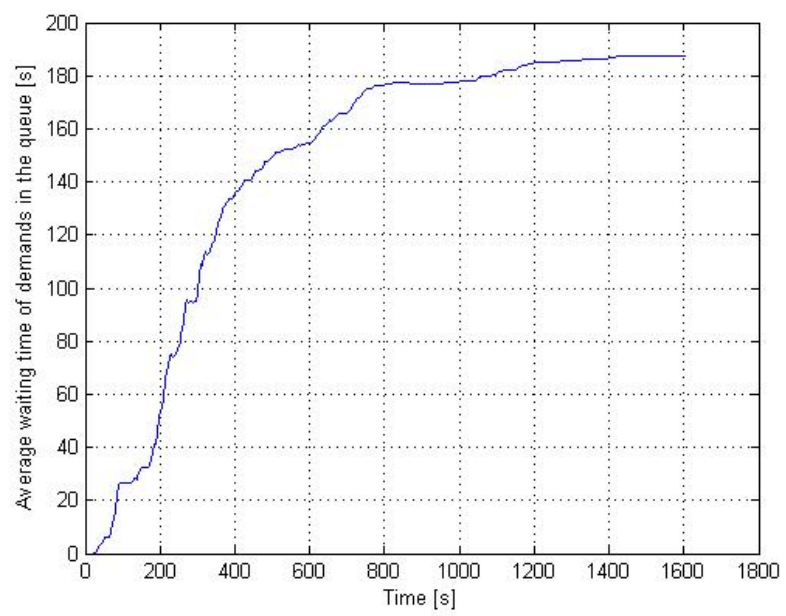

Figure 2. Input: average waiting time of demands in the queue of demands

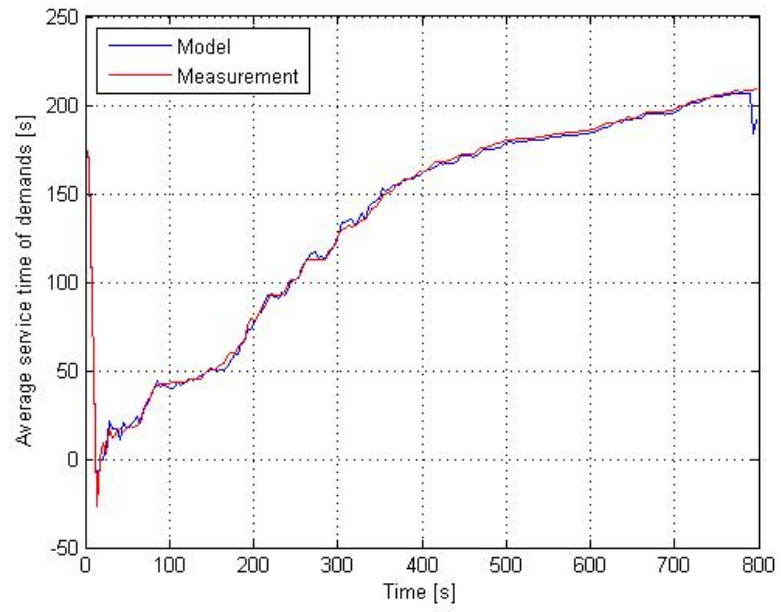

Figure 3. Output of the estimated model. Among 1600 input-output pairs 800 were used for model estimation. 


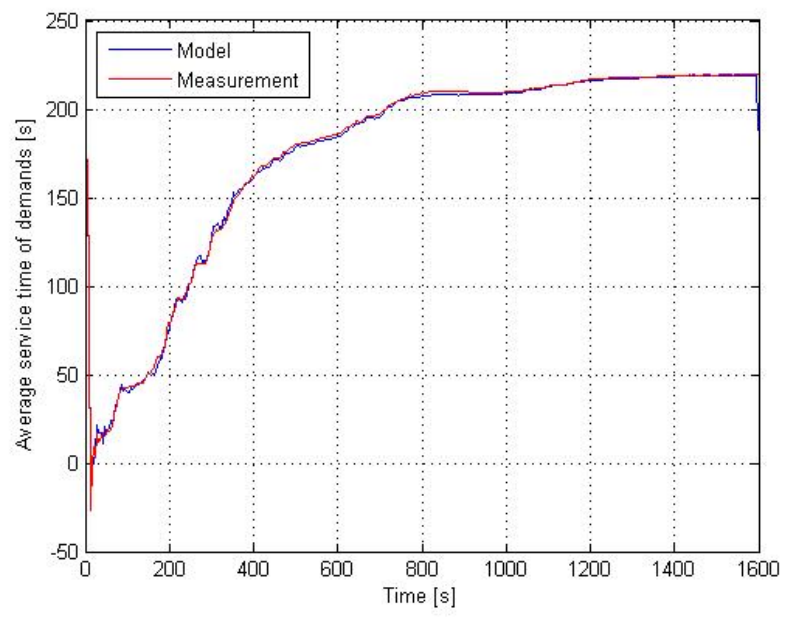

Figure 4. Validation of the estimated model. 1600 input-output pairs were used for validation.
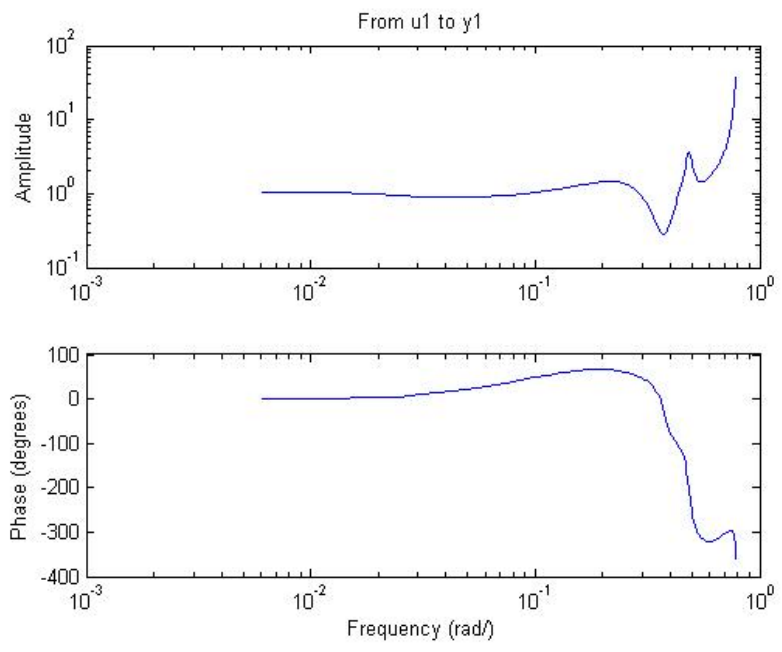

Figure 5. Bode plot of the identified model.

The response of the identified model clearly follows the required characteristics (see 
Fig. 3). The verification of the estimated model has been performed by using 1600 data pairs among which the first 800 has been used for model estimation. The model clearly follows the measured output (see Fig. 4). The frequency response of the system can be followed in Fig. 5. As shown in the next example, the efficiency of identification of queuing systems on subspace basis is strongly influenced by delays present in the system.

\subsection{Example 2}

This example is aimed to show the influence of response time on the accuracy of the identified model. The architecture of the system used in this example is the same as in Example 1. However in contrast to the previous example here the transport time of goods have been doubled, while the number of transportation vehicles was significantly decreased in order to cause longer response times in the system (see Table 2).

The input represented by the average waiting time of demands in the queue of incoming demands can be followed in Fig. 6. The output represented by the average response time together with the response of the estimated model can be followed in Fig. 7. Finally the verification of the model is shown by Fig. 8. Compared to the previous example here the identification yielded a less accurate model (the cause is related to longer waiting times in the system). Although oscillations can be observed, the main characteristics of the measurement is clearly followed by the response of the estimated model. The matrices of the identified state space model are as follows: The estimated system matrix is:

$$
\mathbf{A}=\left[\begin{array}{rrrrrrrr}
0.84984 & 0.10055 & 0.20797 & -0.1294 & 0.17411 & -0.0036611 & 0.022475 & -0.21601 \\
-0.38785 & 0.2428 & -0.018592 & -0.35352 & 0.63397 & 0.001635 & -0.056575 & -0.50946 \\
0.1157 & 0.24374 & 0.21441 & 0.1744 & 0.66165 & 0.27477 & -0.52125 & 0.2711 \\
0.031104 & 1.0741 & -0.085068 & 0.032735 & -0.7941 & 0.14308 & 0.12814 & 0.18568 \\
0.20192 & 0.079581 & -0.67947 & -0.1361 & 0.35766 & -0.11025 & 0.18476 & 0.54493 \\
-0.042457 & -0.38714 & 0.10683 & -0.092881 & -0.14477 & 0.69397 & -0.11695 & 0.16482 \\
-0.013259 & 0.072086 & 0.11943 & 0.046148 & 0.1385 & 0.41882 & 0.77311 & 0.076762 \\
0.13265 & 0.092821 & -0.26987 & -0.31781 & -0.15004 & 0.095917 & 0.012899 & -0.24774
\end{array}\right]
$$

The estimated input matrix is:

$$
\mathbf{B}=\left[\begin{array}{llllllll}
-0.075538 & -0.02332 & 0.3248 & -0.49781 & -0.023601 & -0.1238 & 0.037839 & 0.12997
\end{array}\right]^{T}
$$

The estimated output matrix:

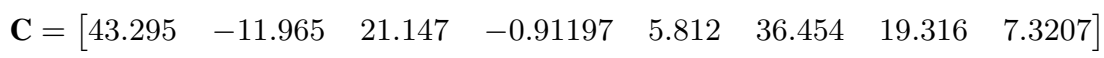

The initial state of the estimated system:

$$
\mathbf{X}(\mathbf{0})=\left[\begin{array}{llllllll}
-2.2054 & 27.437 & 27.061 & -70.7 & -22.441 & -17.067 & 1.1072 & 70.364
\end{array}\right]^{T}
$$


Table 2. Setup of system parameters to generate simulation data

\begin{tabular}{l||l}
\hline Parameter & Value \\
\hline \hline Number of loading machines & 10 \\
Number of vehicles & 2 \\
Number of unloading machines & 3 \\
Queue length of loading machines & 40 \\
Queue length of unloading machines & 40 \\
Queue length of vehicles waiting for loading & 40 \\
Queue length of incoming demands & 50 \\
Queue length of arrived vehicles & 40 \\
Loading time & Exponential distribution with mean 10 \\
Unloading time & Exponential distribution with mean 10 \\
Transport time & Exponential distribution with mean 20 \\
Return time of vehicles & Exponential distribution with mean 10 \\
Incoming rate of demands & Exponential distribution with mean 1 \\
\hline
\end{tabular}

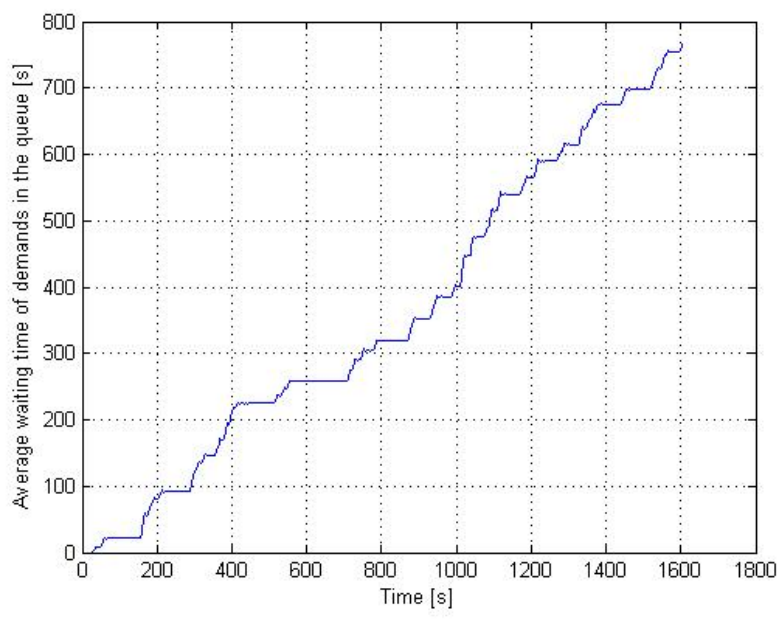

Figure 6. Input: average waiting time of demands in the queue of demands 
T. Vadvári and P. Várlaki - Acta Technica Jaurinensis, Vol.10., No.2., pp. 84-98, 2017

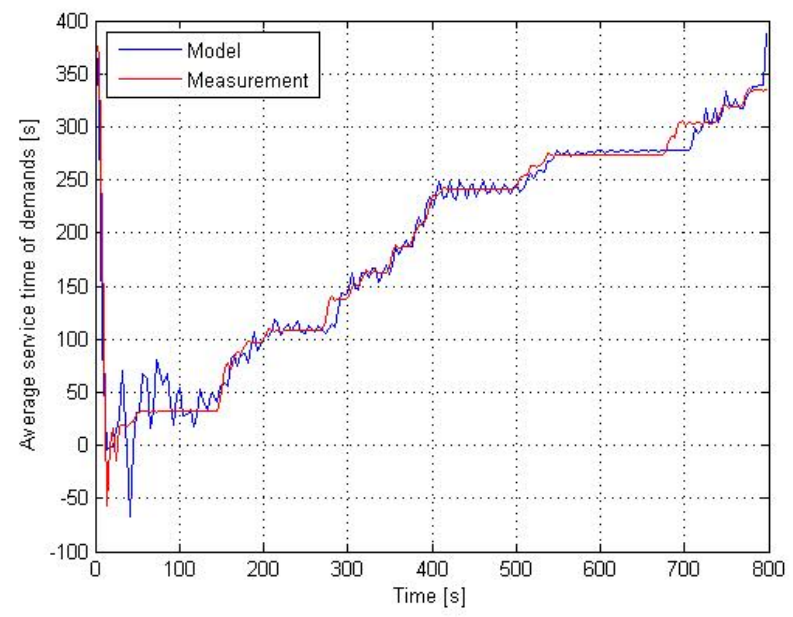

Figure 7. Output of the estimated model. Among 1600 input-output pairs 800 were used for model estimation.

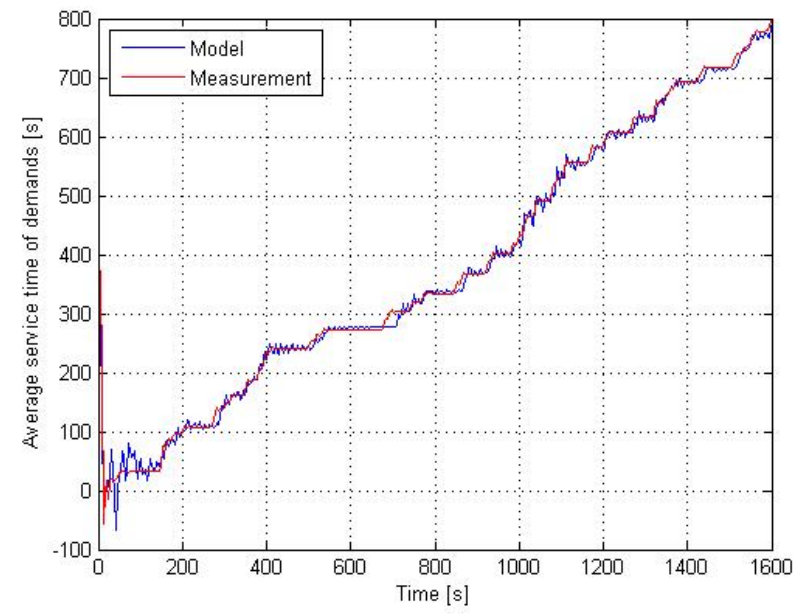

Figure 8. Validation of the estimated model. 1600 input-output pairs were used for validation. 

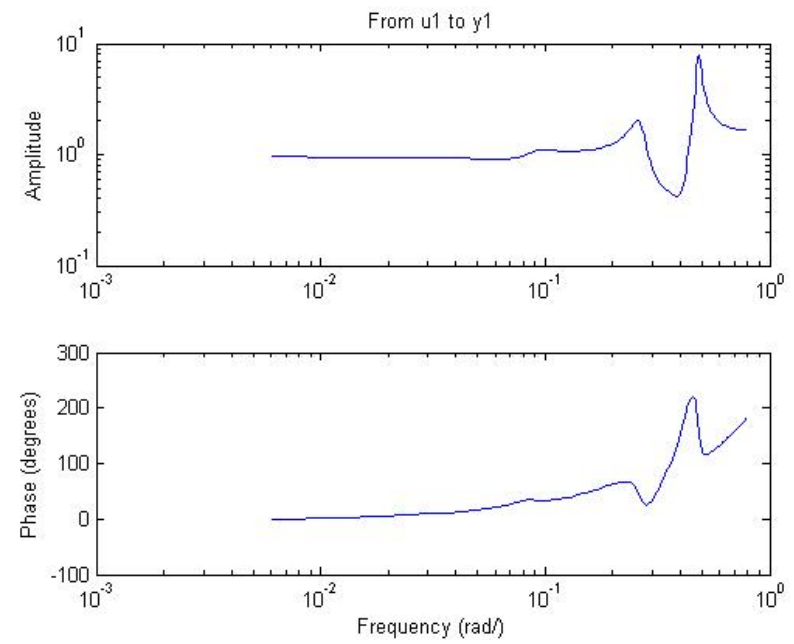

Figure 9. Bode plot of the identified model.

It can be recognized that the accuracy of the identified model is negatively affected by longer response times (compared to the previous example) in the queuing system. This inaccuracy appears in form of oscillations along the main characteristics of the system response (see Fig. 7). However the main characteristic of the model response are kept close to the measured one, thus crucial delays in the system may be detected even by evaluating the response of the identified model. The verification of the estimated model has been performed by using 1600 data pairs among which the first 800 has been used for model estimation. The main characteristics of the model clearly follows the measured output (see Fig. 8). The frequency response of the system can be followed in Fig. 9.

\section{Conclusions}

During our previous studies we have investigated state space models and subspace identification to model and identify supply chains or loading systems as well as to characterize the dependency between its certain factors. In this paper we have turned the focus on the impact of delays on the accuracy of the identified model. Through simulations it was shown that increasing the delays in the system causes oscillations along the main characteristics of the estimated response. The loading systems have been modeled by discrete time, linear, time-invariant state space models and identified on subspace basis. The input-output data used for identification have been generated by a simulated loading system implemented in Matlab Simulink framework. 


\section{References}

[1] K. Tang, C. Yang and J. Yang, "A Supply Chain Network Design Model for Deteriorating Items,"International Conference on Computational Intelligence and Security, Harbin, doi: 10.1109/CIS.2007.140, pp. 1020-1024, 2007.

[2] H. L. Yin, "A New Method for Supply Chain Optimization with Facility Fail Risks,"9th International Conference on Computational Intelligence and Security, Leshan, doi: 10.1109/CIS.2013.81, pp. 353-357, 2013.

[3] V. Bhaskar, P. Lallement, "Modeling a Supply Chain Using a Network of Queues,'Applied Mathematical Modelling, Vol. 34, Issue 8, ISSN 0307-904X, http://dx.doi.org/10.1016/j.apm.2009.10.019, pp. 2074-2088, August 2010.

[4] I. Harmati, G. Orbán, P. Várlaki, "Takagi-Sugeno Fuzzy Control Models for Large Scale Logistics Systems, " Proc. Computational Intelligence and Intelligent Informatics International Symposium (ISCIII 07), Marocco, Agadir, doi: 10.1109/ISCIII.2007.367389, pp. 199-203, March 2007.

[5] G. Orbán, P. Várlaki, "Fuzzy Modelling for Service Strategy and Operational Control of Loading Systems, "Acta Technica Jaurinensis, Series Logistica, Vol. 2, No. 3, Published by Széchenyi István University, Faculty of Engineering Sciences, ISSN 1789-6932, pp. 375-391, 2009.

[6] I.Á Harmati, L.T. Kóczy, "On the Sensitivity of Weighted General Mean Based Type2 Fuzzy Signatures, ”In: Rutkowski L., Korytkowski M., Scherer R., Tadeusiewicz. R., Zadeh L., Zurada J. (eds) Artificial Intelligence and Soft Computing. ICAISC 2016. Lecture Notes in Computer Science, doi:10.1007/978-3-319-39378-0_19, pp. 206-218, June 12-16, 2016.

[7] I.Á Harmati, L.T. Kóczy, “On the Sensitivity of Type-2 Fuzzy Signatures and the Generalizations of the Extension Principle, "2016 IEEE International Conference on Fuzzy Systems, Vancouver, BC, doi:10.1109/FUZZ-IEEE.2016.7737839, pp. 13011307, July 24-29, 2016.

[8] Jing-Shing Yao, Feng-Tse Lin, "Constructing a Fuzzy Flow-Shop Sequencing Model Based on Statistical Data, "International Journal of Approximate Reasoning, Vol. 29, Issue 3, ISSN 0888-613X, doi:10.1016/S0888-613X(01)00064-0, pp. 215-234, March 2002.

[9] P.V. Sevastjanov, P. Róg, "Fuzzy Modeling of Manufacturing and Logistic Systems," Mathematics and Computers in Simulation, Vol. 63, Issue 6, ISSN 0378-4754, doi:10.1016/S0378-4754(03)00064-8, pp. 569-585, 24 November 2003.

[10] T. Vadvári, P. Várlaki, "Queuing Models and Subspace Identification in Logistics,"Acta Technica Jaurinensis, Vol. 8, No. 1, pp. 63-76, 2015. 
[11] A. Gunasekaran, C. Patel, Ronald E. McGaughey, "A Framework for Supply Chain Performance Measurement,"International Journal of Production Economics, Vol. 87, Issue 3, ISSN 0925-5273, doi:10.1016/j.ijpe.2003.08.003, pp. 333-347, 18 February 2004.

[12] Peter Van Overschee, Bart De Moor, "Subspace Identification for Linear Systems, Theory - Implementation - Applications, "Kluwer Academic Publishers, Boston/London/Dordrecht, ISBN: 978-1461380610, p. 272, 2011.

[13] L. Szeidl, P. Baranyi, Z. Petres, and P. Várlaki, "Numerical Reconstruction of the HOSVD Based Canonical Form of Polytopic Dynamic Models,"3rd International Symposium on Computational Intelligence and Intelligent Informatics, Agadir, Morocco, doi:10.1109/INES.2006.1689368, pp. 111-116, 2007.

[14] S. Nagy, Z. Petres, and P. Baranyi, “TP Tool-a MATLAB Toolbox for TP Model Transformation "in Proc. of 8th International Symposium of Hungarian Researchers on Computational Intelligence and Informatics, Budapest, Hungary, pp. 483-495, 2007.

[15] P. Baranyi, Z. Petres, P. Korondi, Y. Yam, H. Hashimoto, “Complexity Relaxation of the Tensor Product Model Transformation for Higher Dimensional Problems,"Asian Journal of Control, Vol. 9, No. 2, pp. 195-200, 2007, doi: 10.1111/j.19346093.2007.tb00323.x

[16] P. Baranyi, D. Tikk, Y. Yam, R. J. Patton, "From Differential Equations to PDC Controller Design via Numerical Transformation,'Computers in Industry, Vol. 51, No. 3, doi:10.1016/S0166-3615(03)00058-7, pp. 281-297, 2003.

[17] P. van Overschee, B.L. de Moor, "Subspace Identification for Linear Systems: Theory - Implementation - Applications, "Springer, Softcover reprint of the original 1st ed. 1996 edition, October 8, 2011. 